\title{
Investigation of a novel nanothermite colloid based on CuO coated CNTs on the thermo-analytical characteristics of 1,3,5-trinitro-1,3,5-triazinane
}

ahmed elbeih ( $\nabla$ elbeih.czech@gmail.com )

military technical college

\section{Research Article}

Keywords: Electroless plating, Nanoparticles, Multi-walled carbon nanotubes (MWCNTs), nanothermites, Explosive

Posted Date: August 4th, 2021

DOl: https://doi.org/10.21203/rs.3.rs-778281/v1

License: (c) (1) This work is licensed under a Creative Commons Attribution 4.0 International License.

Read Full License 


\title{
Investigation of a novel nanothermite colloid based on $\mathrm{CuO}$ coated CNTs on the thermo-analytical characteristics of 1,3,5-trinitro-1,3,5- triazinane
}

\author{
Ahmed K. HUSSEIN ${ }^{1}$, Mohamed G. ZAKI ${ }^{1}$, Ahmed ELBEIH ${ }^{1^{*}}$ \\ Military Technical College, Kobry Elkobbah, Cairo, Egypt \\ *Corresponding author: elbeih.czech@gmail.com - elbeih.ahmed@mtc.edu.eg
}

\begin{abstract}
:
Nanothermite colloid is a promising field of research used to enhance the characteristics of energetic materials. In this study, pre-treated surfaces of multiwall-carbon nanotubes (MWCNTs) were catalysed to help the deposition of a metal and the coating process by copper $(\mathrm{Cu})$ nanoscale layer through electroless deposition. The formed hybrids coated by $\mathrm{Cu}$ were treated at $250^{\circ} \mathrm{C}$ to form MWCNTs coated by $\mathrm{CuO}$. Isopropyl alcohol was used to suspend the coated MWCNTs with aluminium nanoparticles $(120 \mathrm{~nm})$ in order to form nano thermite colloid by ultrasonic technique. The presence of $\mathrm{CuO}$ layer plays the role of an active oxidizer for the Aluminum nanoparticles. The obtained colloid was incorporated and dispersed in 1,3,5-trinitro-1,3,5-triazinane (RDX). The influence of colloid on RDX decomposition kinetic was evaluated using the isoconversional methods (modified KissingerAkahira-Sunose (KAS) methods). The mean value of apparent activation energy was reduced by $37.5 \%$. This dramatic change in RDX decomposition ascribed trait to the nano-thermite colloid reactivity and the facile integration of colloidal thermite particles with the RDX.
\end{abstract}

Keywords: Electroless plating, Nanoparticles, Multi-walled carbon nanotubes (MWCNTs), nanothermites, Explosive.

\section{Introduction:}

The energy output of high explosives can be increased by raising the contribution of the heat of explosion $[1,2]$. The reactive metal particles might raise the energy density with increasing the total impulse of explosives[3, 4]. Aluminum with its variable shapes and sizes has always been an area of interest when used with metal oxides and incorporated in energetic formulations to increase the overall energy and performance $[5,6]$.

Thermite reactions (metal oxide/metal) have high exothermicity and propagate based on their sustained oxygen content; these reactions find wide applications in high energy systems. On the other side, nano-thermites have other various applications especially in the devices used to 
generate energy in the application of modern energetic materials (MEMs) [3]. Since multiwall carbon nanotubes (MWCNTs) offer relatively large surface areas, they are effective carriers for nanothermite particles.

For pure ordinary organic explosives; the explosion heat depends on the combustion heat produced from the constituent fuel elements on the material (carbon and hydrogen)[7]. Simply the combustion heat could be increased by adding fuels (with high heat of combustion) to the explosive to enhance the explosive overall energy [8], [9]. Most traditional energetic materials might produce free oxygen in the plasma phase of the explosion. The presence of oxidizer (such as thermites or hybrid materials containing sufficient oxidizer) could enhance the performance of the energetic materials due to the availability of oxygen inside the matrix itself $[10,11]$. Thermites are composed of fuel and oxidizer which are able to undergo reduction-oxidation (Redox) reaction and caused the oxidation reaction to produce a stable oxide and free metal from the reduction process of the metallic oxide. The reaction has high exothermicity and propagates by the self-sustaining oxygen content. Consequence of the released heat and the produced temperature from the thermite reactions, thermites have several applications in welding, reactive fragments and ordinance disposal [12]. Due to the redox reaction, the formed metal might tolerate further combustion in the availability of more oxygen under high temperature and generate more heat [9].

A comparison of the volumetric and gravimetric heats of reaction of the thermite/redox reaction with that of highly energetic explosives proved that the thermite reactions produce higher energies [13] as shown in Fig.1.

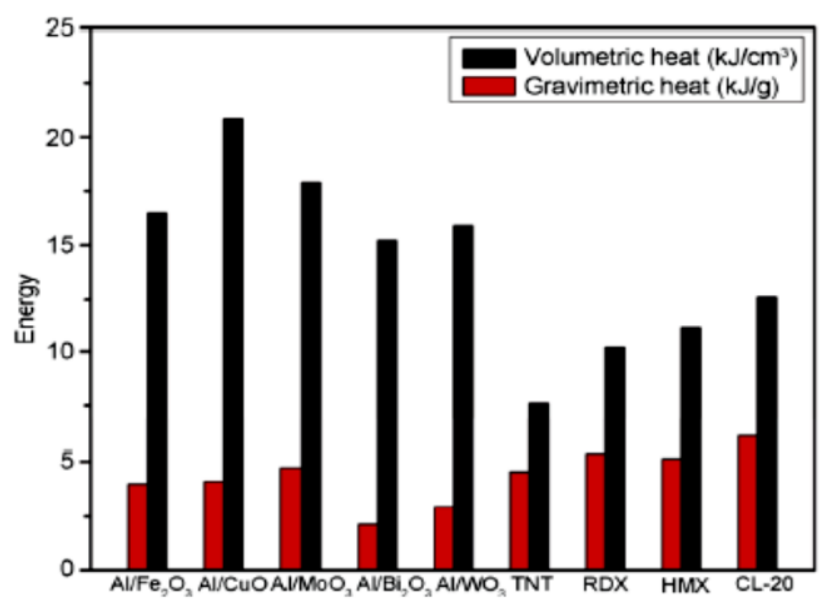

Fig 1: Comparing the produced energy from thermites composition with common explosives 
One of the advantages of nanotechnology is the great decreasing of the material particle size and increasing the ratio of surface/volume as shown in Fig. 2. Consequently, the highly increase in the surface energy causes improvement of the contact area and mixing homogeneity [14].
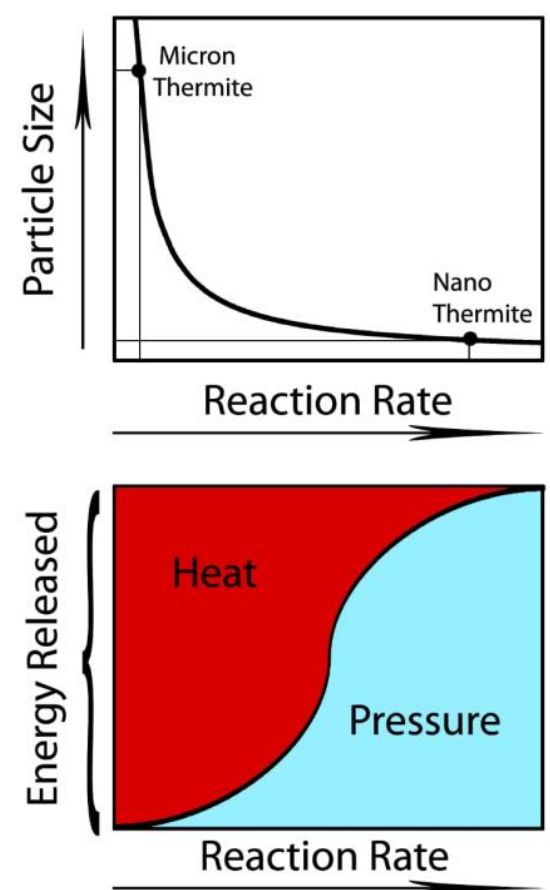

Fig. 2 Effect of reactants particle sizes on the reaction rate and released energy [15]

All these properties resulted in significant enhancement of the reactivity and increasing the propagation of the reaction rate. The enhancement of nano-composite thermite reactivity is resulted from the high connection of $\mathrm{Al}$ with the oxidizer (metal oxide) [16].

Multi-walled-carbon nanotubes (MWCNTs) contain a structure with rich pore and large surface area $\left(>700 \mathrm{~m}^{2} / \mathrm{g}\right)$, which might be used as catalyst $[17,18]$. If MWCNTs were functionalized with energetic groups, compounds or metal fuels, they become more energetic and might find applications in highly energetic systems, fine welding, aircraft ejection seats and nanothermite synthesis [19]

This superior surface area of multiwall-carbon nanotubes (MWCNTs) can be employed in synthesis of hybrid materials by plating the nanotubes with different metals to form a nanocomposite. The metallization process depends on the surface catalyzation of MWCNTs, which increases the activity of the surface to enhance the bonding of the nanotubes with the deposited metal [20-22]. 
The metallization process of MWCNTs could be carried out by an electroless deposition process at room temperature. Electroless deposition technique [17] is the preferred technique when it is compared with other techniques such as molecular-level mixing [23], and chemical/physical vapour deposition [24] due to its simplicity and high efficiency where it is used for the deposition of different elements such as copper[21], nickel [25], silver, gold [26], and cobalt onto MWCNTs [27].

MWCNTs should be pretreated by acid to purify and catalyze the surface of MWCNTs in order to be ready for the covering process, as shown in schematic representation in Fig. 3.

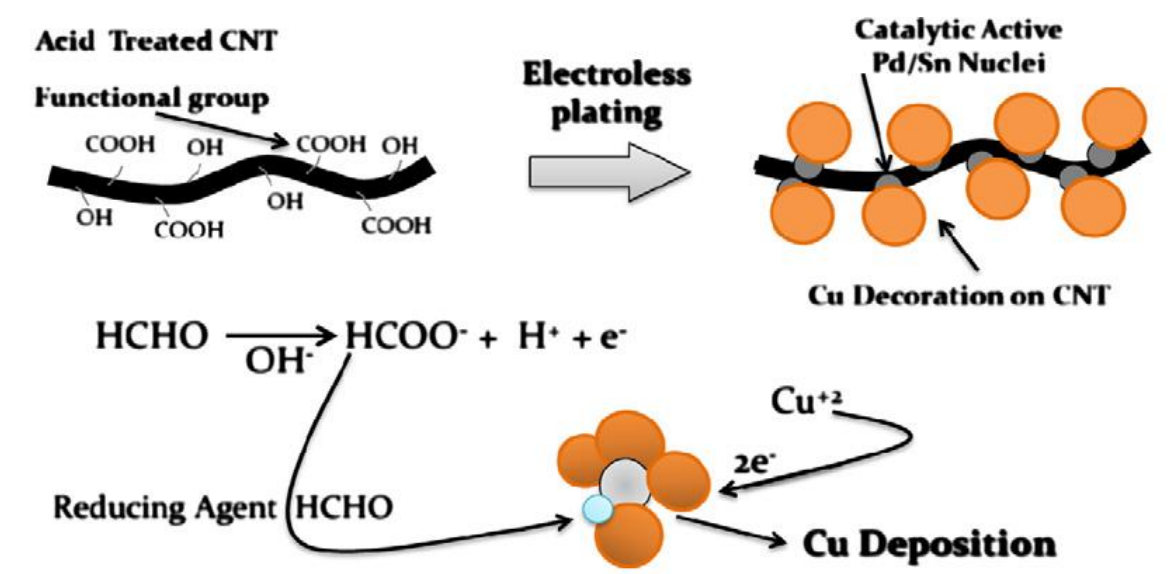

Fig. 3: A scheme for the electroless plating of MWCNTs by copper. [28]

An effective plating bath containing formaldhyde as a reducing agent was designed to be used for the $\mathrm{Cu}$ deposition on MWCNTs. The process is achieved by the reaction of the functional groups of MWCNTs surfaces with the metal ions. The particles of $\mathrm{Cu}$ are deposited on the pretreated surface of MWCNTs with uniform distribution [28].

This investigation reports on the synthesis of Cu-MWCNTs hybrid nanomaterials by electroless plating, then the complete annealing process at $\left(250{ }^{\circ} \mathrm{C}\right)$ to get $\mathrm{CuO}-\mathrm{MWCNTs}$. The weight ratio of $\mathrm{Cu}$ : MWCNTs affects the thickness of the $\mathrm{Cu}$ layers deposited on the MWCNTs and its morphology.

Nano-composite manufacture is based on the dispersion of CuO-MWCNTs in acetone with aluminum nanoparticles. The acetone acts as dispersion medium and solvent for the energetic system to enhance the nano-particle distribution in the medium.

The synthesized energetic hybrid material (MWCNTs/CuO/Al) produces high amount of gaseous where the produced $\mathrm{Cu}$ metal might be converted to gas as a result of its low boiling point $\left(1000{ }^{\circ} \mathrm{C}\right)[29]$. While the conversion process takes place, MWCNTs/CuO/Al reactions will proceed with high rate. 
RDX (1,3,5-trinitro-1,3,5-triazinane) is one of the most usable explosives all over the world [30], it has several applications such as production of plastic explosives (composition C-4 and semtex-1H) [31, 32], highly pressed explosive for shaped charges and formation of demolition charges $[33,34]$. In this paper, hybrid material based on $\mathrm{CuO}$ plated MWCNTs was synthesized by electroless plating process and employed with nano $\operatorname{Al}(100 \mathrm{~nm})$ to form a hybrid thermite material. The influence of the prepared nano-thermite mixture on the thermoanalytical characteristics of RDX was discussed. This study presents a novel process for the development of a hybrid thermite materials and their incorporation with the explosives for several applications in the field of energetic materials.

\section{Experimental}

\subsection{Materials}

MWCNTs were obtained from US research nanomaterials, Inc. (purity of 95\%, 5-10 nm inside diameter, 20-30 $\mathrm{nm}$ outside diameter and 0.5-2 $\mu \mathrm{m}$ length). Also copper(II) sulfate penta-hydrate $\left(\mathrm{CuSO}_{4} \cdot 5 \mathrm{H}_{2} \mathrm{O}\right.$, Sigma-Aldrich), Sodium Citrate $\left(\mathrm{C}_{6} \mathrm{H}_{5} \mathrm{Na}_{3} \mathrm{O}_{7} \cdot 2 \mathrm{H}_{2} \mathrm{O}\right.$, Bio shop), sodium hydroxide $(\mathrm{NaOH}, \mathrm{CALEDON}$ Laboratory Chemicals), Hydrochloric acid $(\mathrm{HCl}$, CALEDON), Acetone (99.9\% Penta chemicals), Stannous chloride $\left(\mathrm{SnCl}_{2}\right.$, CALEDON Laboratory Chemicals), stannous chloride dihydrate $\left(\mathrm{SnCl}_{2} \cdot 2 \mathrm{H}_{2} \mathrm{O}, 98 \%\right.$, Caledon), palladium (II) chloride ( $\mathrm{PdCl}_{2}$, Art-craft chemicals Inc.) were used. In addition, RDX (Dyno Nobel, mixture of Class 2 and 5) was used.

\subsection{MWCNTs Catalyzation process}

Catalyzation of MWCNTs is a surface pre-treatment procedure which can be employed to deposit different metals on the surface. Catalyzation process encompasses two main stages including sensitization and activation as demonstrated in Fig. 4 [35-37].

Sensitization is the initial process to create active sites on the structure of MWCNTs using $\mathrm{SnCl}_{2}$ and strong acid. Sensitization mixture composed of $4 \mathrm{~g} \mathrm{SnCl}_{2}, 20 \mathrm{ml}$ conc. $\mathrm{HCl}$, and $180 \mathrm{ml}$ deionized water. $4 \mathrm{~g}$ of MWCNT was dispersed in the mixture and sonicated with ultra-sonic probe sonicator (Qsonica - Q500) for an hour. As a result, a catalyzed surface should be effectively created. The sensitized MWCNTs were filtrated and washed by water then it was dried at $75{ }^{\circ} \mathrm{C}$ for one hour under vacuum.

Sensitized MWCNTs was activated with $200 \mathrm{ml}$ activating reagent $\left(0.06 \mathrm{~g} \mathrm{PdCl}_{2}, 4 \mathrm{ml} \mathrm{HCl}\right.$, and $200 \mathrm{ml}$ dionized water). Activation process was achieved under sonication process for 340 minutes. The activated MWCNTs was finally filtered and washed by dionized water then dried. 


\subsection{Metallizing the MWCNTs by Copper}

Electroless plating of MWCNTs was performed by the deposition of copper nano-crystals to get 16 fold of $\mathrm{Cu}$ to 1 fold nano-carbon materials [35, 38, 39]. This emerging path could present continual $\mathrm{Cu}$ surface layer. The activated nano-carbons (0.6 gram) were scattered in $400 \mathrm{ml}$ of the plating solution. The solution contains $37 \mathrm{~g}$ of $\mathrm{CuSO}_{4} .5 \mathrm{H}_{2} \mathrm{O}, 88 \mathrm{~g}$ $\mathrm{Na}_{3} \mathrm{C}_{6} \mathrm{H}_{5} \mathrm{O}_{7} \cdot 2 \mathrm{H}_{2} \mathrm{O}, 110 \mathrm{ml}$ of $\mathrm{HCHO}$, and deionized water. Ultrasonic Probe was applied to the MWCNTs nano-carbons materials for one hour. During the sonication, $1000 \mathrm{ml}$ of sodium hydroxide solution was supplied to maintain the $\mathrm{pH}$ at 9:10. The complete coating of MWCNTs with $\mathrm{Cu}$ was observed by changing the black colour of the slurry to red brownish. The prepared sample was filtered and washed by deionized water, then left for 6 hours under heating at $260{ }^{\circ} \mathrm{C}$ to get MWCNTs coated by $\mathrm{CuO}$. The complete MWCNTs metallization and annealing process are shown in Fig. 4.

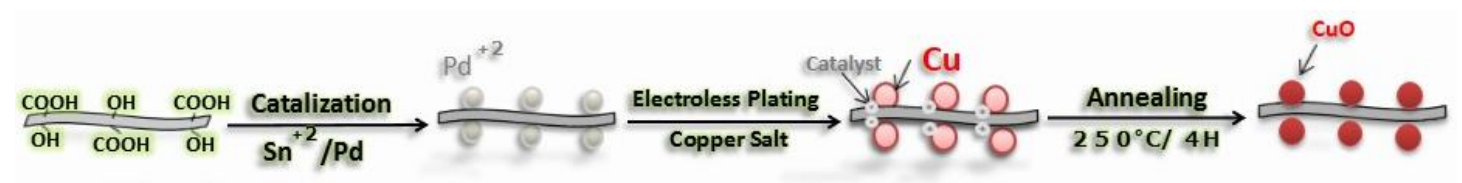

Fig. 4: Schematic diagram for electroless plating methodology of nano-carbons/Cu.

\subsection{Characterization of MWCNT.}

The crystal morphology of MWCNTs and CuO/MWCNTs was studied by TEM (JEM-2010F by Joel Corporation). The crystallinity of the samples was determined by using X-ray diffraction (XRD) based on Bruker D8 Discover instrument. Also, SEM (ZEISS SEM EVO 10 MA) was used to study the crystal sizes and shapes of the studied samples.

\subsection{Dispersion of CuO-MWCNTs with Alex into energetic matrix}

CuO-MWCNTs/Al/RDX was developed by solvent blending approach. The following procedure was applied: The calculated amounts of 94\% RDX, 1\% CuO-MWCNTs and 5\% $\mathrm{Al}$ were weighed accurately. CuO-MWCNTs was added with aluminium nanoparticles (Alex) in acetone placed on ultrasonic path in order to dissipate the oxidizer and fuel particles and eliminate any aggregation. The prepared nanothermite was added to the completely dissolved RDX in acetone under sonication followed by gradual heating to guarantee the evaporation of acetone and complete dispersion of nanothermite particles with the energetic matrix (RDX).

\subsection{Thermal analysis study}

The RDX/MWCNT/Al/CuO and the pure RDX were studied by Thermogravimetric analysis (TGA55, TA Instruments, USA, open high temperature platinum crucible) at heating rates of 
3, 5, 7 and $10{ }^{\circ} \mathrm{C} \min ^{-1}$. The samples were tested in a range of temperature of $40-350{ }^{\circ} \mathrm{C}$, and the mass of the sample was 1-2 $\mathrm{mg}$ tested in dynamic nitrogen atmosphere $\left(40 \mathrm{ml} \mathrm{min}^{-1}\right)$.

\subsection{Theoretical investigation}

The decomposition kinetics are based on determining the activation energy, Ea, with the preexponetial factor, $A$, in addition to the model of the reaction, $f(\alpha)$, which are known as kinetic triplet. The evaluation of the solid phase reactions could be investigated by various techniques [40]. Different isothermal or nonisothermal methods could be applied to obtain the decomposition kinetics.

Isoconversional method could be investigated depending on the change of the rate of process

$$
\frac{d \alpha}{d t}=k(T) f(\alpha)
$$

In this case, the reaction model is $f(\alpha)$ and $\mathrm{k}(\mathrm{T})$ is a rate constant (depend on the temperature) and could be determined by applying Arrhenius equation

$$
k(T)=\operatorname{Aexp}\left(\frac{-E}{R T}\right)
$$

By adding eq. 2 to eq.1, the kinetic differentiation could be:

$$
\frac{d \alpha}{d t}=\operatorname{Aexp}\left(\frac{-E}{R T}\right) f(\alpha)
$$

By the integration of eq.3, the following equation was obtained

$$
g(\alpha) \equiv \int_{0}^{\alpha} \frac{d \alpha}{f(\alpha)}=A \int_{0}^{t} \exp \left(\frac{-E}{R T}\right) d t
$$

Here; $g(\alpha)$ represents the integrated order for the model of the reaction.

In the iso-conversional method, the model of the reaction is based on Eq. 1 and does not depend on the temperature. In this study, the modified Kissinger-Akahira-Sunose (KAS) methods were investigated:

$$
\ln \left(\frac{\beta_{\mathrm{i}}}{\mathrm{T}_{\alpha, \mathrm{i}}^{1.92}}\right)=\text { const }-1.0008\left(\frac{\mathrm{E}_{\alpha}}{\mathrm{RT}_{\alpha}}\right)
$$

The symbol $T_{p}$ represents the exothermic peak temperature at the selected rates and $\beta$ is the selected heating rate. By plotting of $\ln \left(\frac{\beta_{\mathrm{i}}}{\mathrm{T}_{\alpha, \mathrm{i}}^{1.92}}\right)$ against $\left(\frac{1}{T_{\alpha}}\right)$ (at various heating rate), the slope of the obtained straight line represents the activation energy [41]. 


\section{Results and Discussions}

\subsection{CuO-MWCNTs characteristics}

TEM images of hybrid nancomposite CuO-MWCNTs reveal the $\mathrm{CuO}$ nano-particles $(14 \mathrm{~nm}$ particle size) deposition on the CNT walls. Figure 5 confirms the success of the electroless plating methodology to enhance effectively the surface area occupied by the copper oxide. These surface areas with the localized oxidizer sites could support rapid reactions in the presence of Alex metallic fuel.
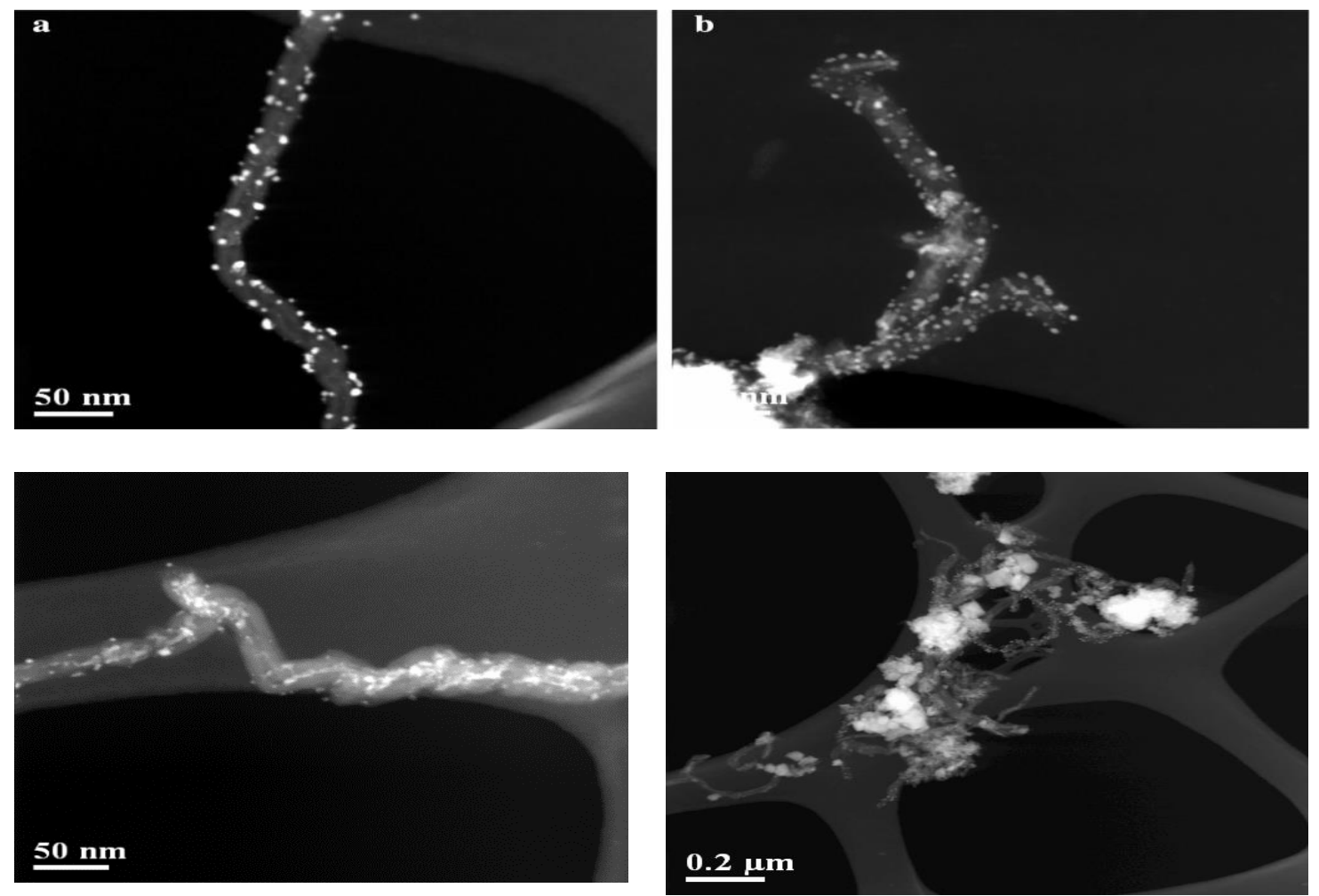

Fig.5 TEM micrographs of CuO/MWNT.

The X-ray diffraction was applied to evaluate the crystallinity of the samples according to the different applied processes. The crystallinity of the synthesized CuO-MWCNTs was examined, as illustrated in Fig. 6. The results indicate that there are ten distinctive peaks of $\mathrm{CuO}$, these peaks are compatible with the data reported on the international centre for diffraction data (JCPDS). 


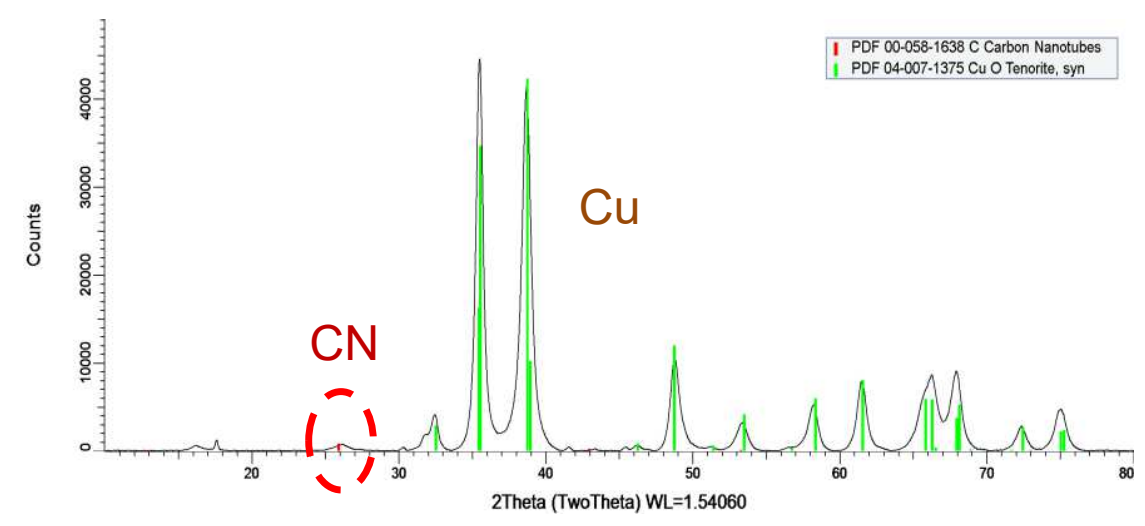

Fig. 6: XRD patterns of CuO-coated MWCNTs

It was observed that there is a high reflection ratio of copper oxide to MWCNTs (16:1) in the XRD patterns, with a high purity (no traces of other materials) of the synthesized hybrid.

The hybrid CuO-MWCNTs morphology was studied using SEM (Fig. 7). The SEM micrographs indicated the effect of the annealing process on the aggregation and agglomeration of hybrid nanoparticles. This challenge has been overcome by the dispersion of CuO-MWCNTs in acetone along with Alex to grow an energetic nanothermite colloid using ultrasonic technique.

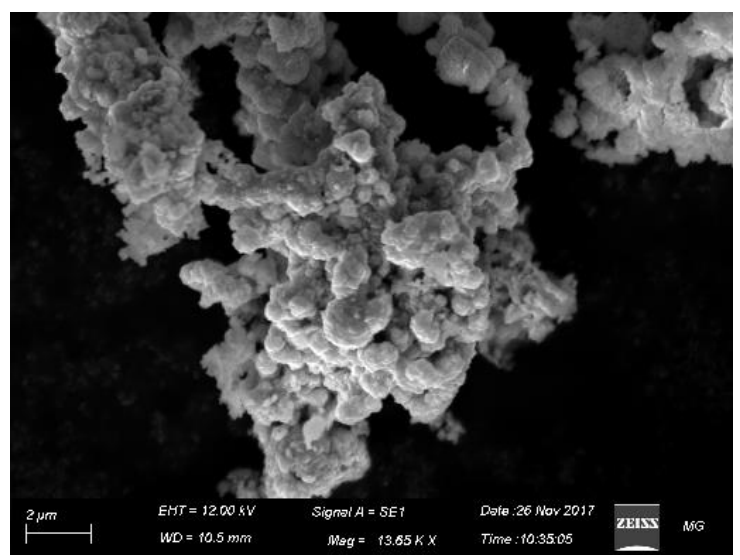

Fig.7 A SEM micrograph of $\mathrm{CuO}$ coated MWCNTs

\subsection{Thermal behavior of the energetic nano composite}

\subsubsection{TG/DTG studies}

In figure 8 , the TG/DTG curves of the pure RDX and the new colloid of CuO-MWCNT-Al nano-thermite at different heating rate were presented and the decomposition data are reported in table 1. It is obvious that the two studied samples have single decomposition process even in the presence of the novel hybrid colloid. In case of $3{ }^{\circ} \mathrm{C} \mathrm{min}{ }^{-1}$ heating rate, the pure RDX started the process of decomposition with onset decomposition temperature of 
$161.2{ }^{\circ} \mathrm{C}$ while the onset decomposition temperature of $\mathrm{RDX} / \mathrm{MWCNT} / \mathrm{Al} / \mathrm{CuO}$ was $168.9^{\circ} \mathrm{C}$. It means that the new colloid CuO-MWCNT-Al has positive influence on the starting of the decomposition process of RDX based colloid. On the other side, the maximum decomposition peak of the pure RDX was observed at $205.6{ }^{\circ} \mathrm{C}$ while it was at $201.9{ }^{\circ} \mathrm{C}$ for the $\mathrm{RDX} / \mathrm{MWCNT} / \mathrm{Al} / \mathrm{CuO}$ nano-thermite. In this case, the maximum decomposition peak of $\mathrm{RDX}$ is higher than the thermite mixture. These results prove that the completion of the decomposition process of RDX/MWCNT/Al/CuO was fast in comparison with the pure RDX. This result might be connected with the high thermal conductivity of both $\mathrm{Al}$ and $\mathrm{CuO}$ which cause quick distribution of the accumulated heat over the high surface area of the sample and speed up the end of the decomposition process. The same phenomena observed at the different studied heating rates, the onset decomposition temperature of $\mathrm{RDX} / \mathrm{MWCNT} / \mathrm{Al} / \mathrm{CuO}$ was higher than $\mathrm{RDX}$ at each individual heating rate but the maximum decomposition peak was lower. These results prove the fast completion of the decomposition process of $\mathrm{RDX} / \mathrm{MWCNT} / \mathrm{Al} / \mathrm{CuO}$ in comparison with the pure RDX. Regarding to the mass loss at the end of the decomposition process, the pure RDX was nearly decomposed completely (the solid sample was converted to gaseous products) with the presence of nearly $1 \%$ residue which might be impurities or non-oxidized carbon soat. In case of $\mathrm{RDX} / \mathrm{MWCNT} / \mathrm{Al} / \mathrm{CuO}$, the remained residue was in the range of 3.4 to $6.2 \%$ which could be due to the residue of the decomposition products of $\mathrm{Al}$ and the $\mathrm{CuO}$ in the colloid.

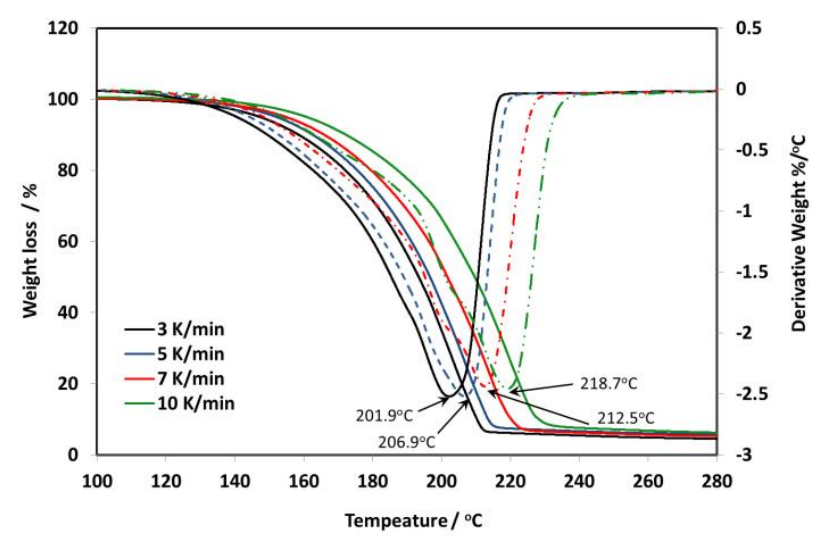

a $\mathrm{RDX} / \mathrm{MWCNT} / \mathrm{Al} / \mathrm{CuO}$

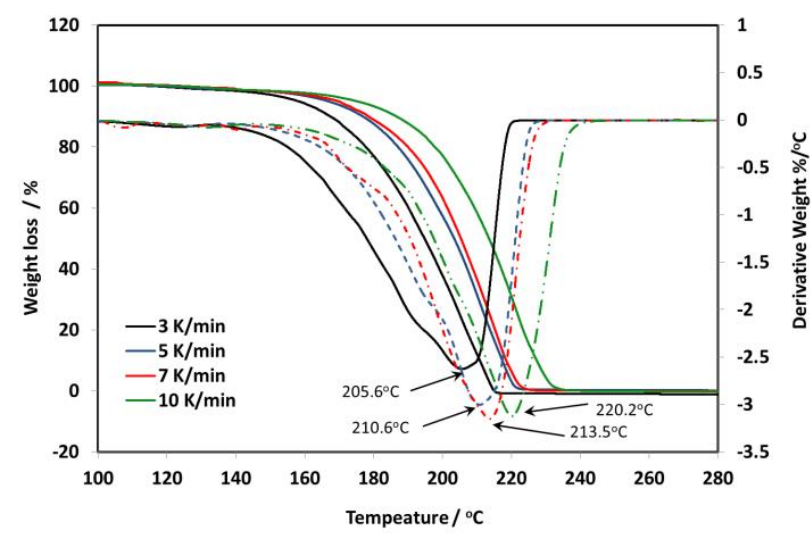

b RDX

Fig. 8 TG/DTG of the prepared colloid and the RDX at the studied heating rates 
Table 1. TG/DTG results of the prepared RDX/MWCNT/Al/CuO-Composite, and the pure RDX at different heating rates

\begin{tabular}{c|ccccc}
\hline \multirow{2}{*}{ type } & \multicolumn{3}{|c}{ TG curve } & \multicolumn{2}{c}{ DTG curve } \\
& $\beta /{ }^{\circ} \mathbf{C} \mathbf{~ m i n}^{-1}$ & $\mathbf{T}_{\mathbf{0}} /{ }^{\mathbf{O}} \mathbf{C}$ & Mass loss $/ \%$ & $\mathbf{T}_{\mathbf{p}} /{ }^{\circ} \mathbf{C}$ & $\mathbf{T}_{\mathbf{o e}} /{ }^{\circ} \mathbf{C}$ \\
\hline \multirow{2}{*}{$\begin{array}{c}\text { RDX/MWCNT/Al/CuO- } \\
\text { Composite }\end{array}$} & 3 & 168.9 & 95.9 & 201.9 & 215.0 \\
& 7 & 170.0 & 93.8 & 206.9 & 217.9 \\
& 10 & 175.3 & 96.6 & 212.5 & 225.0 \\
& 3 & 181.0 & 96.5 & 218.7 & 231.6 \\
\hline \multirow{2}{*}{ RDX } & 5 & 161.2 & 99.9 & 205.6 & 218.5 \\
& 7 & 178.3 & 99.0 & 210.6 & 224.3 \\
& 10 & 181.5 & 98.8 & 213.5 & 225.7 \\
\hline \hline
\end{tabular}

\subsubsection{Non isothermal kinetic parameters studies}

For the advanced modified energetic compositions, it is important to determine the thermal characteristics due to their intrinsic connection with the performance of detonation and combustion of the used composition [42-46]. Also the thermal stability and the activation energy (Ea) are essential to be determined in addition to the decomposition heat [47-51].

Several isoconversional methods can be applied to study the decompositions characteristics of the energetic colloids such as the modified Kissinger-Akahira-Sunose (KAS) which is recommended according to ref. [40]. In this study, KSA is used to determine the Ea at conversion rates ranging from 5 to $90 \%$. In this study, the mean values of $\alpha$ were selected in the interval of 0.25 to 0.85 due to the inaccuracy of the results from the tail peaks. The obtained data are reported in Table 2. The mean values of the obtained $E_{a}$ of the studied samples were 113.5 and $181.5 \mathrm{~kJ} \mathrm{~mol}^{-1}$ for $\mathrm{RDX} / \mathrm{MWCNT} / \mathrm{Al} / \mathrm{CuO}$ and pure RDX respectively. It is obvious that the new colloidal nano-thermite has a dramatic influence on the thermal decomposition kinetics of the energetic filler. The Ea of the RDX colloid was reduced by $37.5 \%$ in comparison with the pure RDX. Yan et al proved that the addition of nanosized particles to the explosives accelerate their decomposition process in comparison with the micro-sized particles due to the large specific surface area (SSA) of the nanoparticles with larger active sites which increase the reactivity of the explosives and decrease their decomposition peak temperature[19]. In case of RDX/MWCNT/Al/CuO, the decomposition reaction is complex and nonlinear in comparison with the pure RDX. It was stated the decomposition mechanism of RDX depends on the grain formation, nucleation and growth of reactive sites[52]. The addition of nano-materials could change the decomposition mechanism of the RDX, causing the modification of the gaseous 
products with different percentages. In addition, the used MWCNTs have high conductivity heat, which caused the enhancement of the conduction heat on the surface of the colloid and could decrease the decomposition temperature. Also the large SSA of MWCNTs could absorb some of the reductive gaseous products and prevent their getting-away from the surface. So, the reaction was continued in the condensed phase and resulted in the improvement of the catalytic effect.

Table 2. Kinetic parameters of RDX/MWCNT/Al/CuO and RDX by modified isoconversional KAS method

\begin{tabular}{|c|c|c|c|c|c|c|}
\hline \multirow[b]{2}{*}{$\alpha$ reacted } & \multicolumn{3}{|c|}{ RDX/MWCNT/Al/CuO } & \multicolumn{3}{|c|}{ RDX } \\
\hline & $\begin{array}{c}\mathrm{E}_{\mathrm{a}} \\
\mathrm{kJ} \cdot \mathrm{mol}^{-1}\end{array}$ & $\begin{array}{c}\log A \\
\mathrm{~s}^{-1}\end{array}$ & $\mathrm{R}^{2}$ & $\begin{array}{c}\mathrm{E}_{\mathrm{a}} \\
\mathrm{kJ} \cdot \mathrm{mol}^{-1}\end{array}$ & $\begin{array}{c}\log A \\
\mathrm{~s}^{-1}\end{array}$ & $\mathrm{R}^{2}$ \\
\hline 0.05 & 150.4 & 20.1 & 0.9593 & 186.2 & 21.0 & 0.9635 \\
\hline 0.10 & 132.1 & 17.4 & 0.9848 & 184.0 & 20.7 & 0.9765 \\
\hline 0.15 & 131.3 & 17.1 & 0.9911 & 182.9 & 20.6 & 0.9865 \\
\hline 0.20 & 130.0 & 16.7 & 0.9608 & 181.8 & 20.4 & 0.9828 \\
\hline 0.25 & 131.3 & 16.7 & 0.9994 & 172.4 & 19.3 & 0.9817 \\
\hline 0.30 & 129.0 & 16.2 & 0.9846 & 171.7 & 19.1 & 0.9828 \\
\hline 0.35 & 122.0 & 15.3 & 0.9951 & 173.4 & 19.3 & 0.9828 \\
\hline 0.40 & 115.0 & 14.4 & 0.9889 & 174.5 & 19.3 & 0.9850 \\
\hline 0.45 & 109.1 & 13.6 & 0.9894 & 174.4 & 19.3 & 0.9882 \\
\hline 0.50 & 115.9 & 14.3 & 0.9892 & 172.5 & 19.0 & 0.9852 \\
\hline 0.55 & 107.4 & 13.3 & 0.9910 & 178.8 & 19.6 & 0.9917 \\
\hline 0.60 & 107.7 & 13.3 & 0.9911 & 182.4 & 20.0 & 0.9932 \\
\hline 0.65 & 107.3 & 13.2 & 0.9911 & 188.9 & 20.6 & 0.9958 \\
\hline 0.70 & 107.5 & 13.1 & 0.9929 & 189.2 & 20.6 & 0.9973 \\
\hline 0.75 & 108.3 & 13.2 & 0.9929 & 189.6 & 20.6 & 0.9973 \\
\hline 0.80 & 109.8 & 13.3 & 0.9935 & 190.9 & 20.7 & 0.9990 \\
\hline 0.85 & 105.0 & 12.7 & 0.9980 & 189.4 & 20.6 & 0.9987 \\
\hline 0.90 & 105.3 & 12.7 & 0.9980 & 190.0 & 20.6 & 0.9987 \\
\hline Mean value & $113.5 \pm 2.9$ & $14.1 \pm 0.42$ & & $181.6 \pm 2.5$ & $20.1 \pm 0.22$ & \\
\hline
\end{tabular}




\subsubsection{Influence of the conversion extend on the kinetic parameters}

In figure 9 , the dependence of the reaction conversion $(\alpha)$ on the temperature of $\mathrm{RDX} / \mathrm{MWCNT} / \mathrm{Al} / \mathrm{CuO}$ and $\mathrm{RDX}$ at the studied heating rates are presented. The curves of $\mathrm{RDX} / \mathrm{MWCNT} / \mathrm{Al} / \mathrm{CuO}$ look similar to the pure RDX in the extent of conversion with a slight shift related to the temperature. This observation confirms the already mentioned role that the presence the nano-thermite has not negative effect on the stability of RDX.

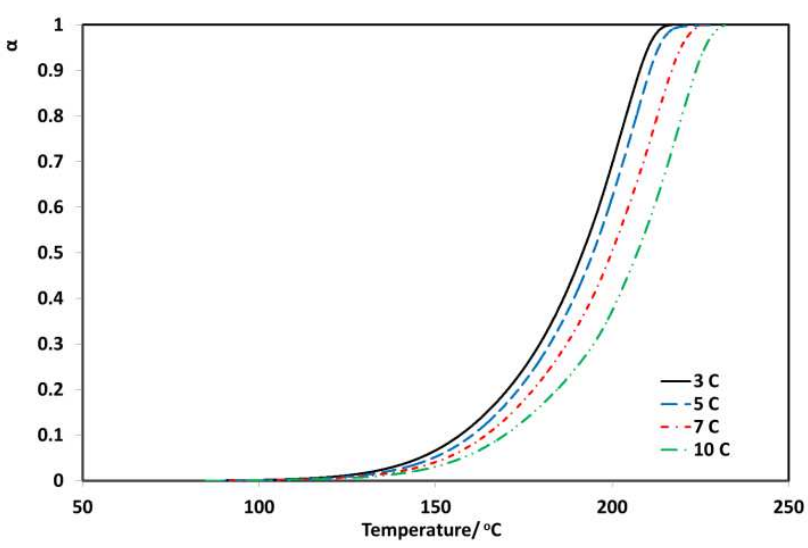

a $\mathrm{RDX} / \mathrm{MWCNT} / \mathrm{Al} / \mathrm{CuO}$

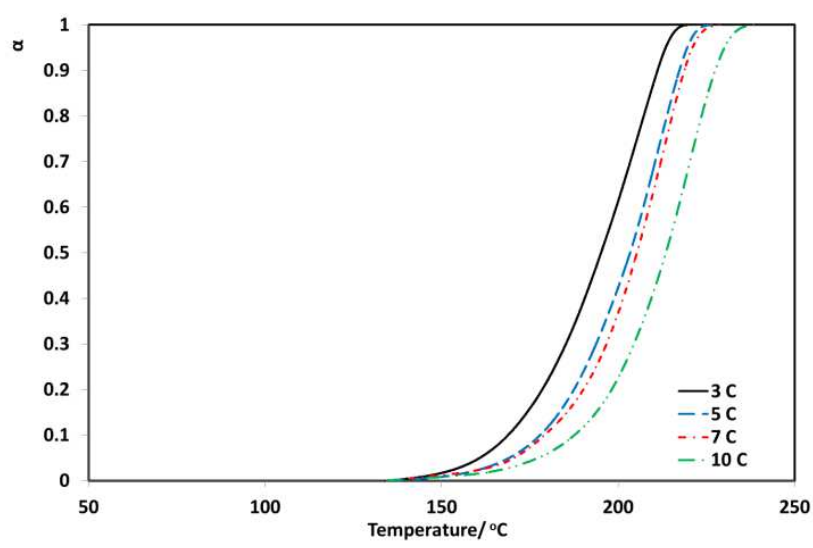

b RDX

Fig. 9 The $\alpha$-T curves for the studied samples at 3,5,7 and 10 heating rate

A dependence of activation energies of RDX/MWCNT/Al/CuO and RDX on the conversion extent are presented in Fig. 10. The activation energy of RDX seems to be constant until extent of conversion of 0.55 , then it starts to increase slightly by approximately $10 \mathrm{~kJ} \mathrm{~mol}^{-1}$. While the RDX/MWCNT/Al/CuO activation energy decreased along with the extent of conversion of 0.55 then started to be on the same value. As mentioned, decreasing the particle size leads to increasing both the specific surface area and the number of surface atoms of the particles which caused strong molecular vibrations of RDX molecules on the surface and increase the molecular energy. It was mentioned that the addition of nanoscale metals or metal oxides, accelerates the decomposition of some explosives due to the improvement of the molecular energy level $[52,53]$. In this study, the RDX molecules on the crystal surface have high energy levels resulted in decreasing the decomposition temperature. Also it was stated by Joseph et al that the presence of nanoscale metal might promote the decomposition of HMX explosive as a result of the physical adsorption applied by the nano-scale metal and the formation of hotspots [54]. This explanation might be suitable to explain the results of RDX (nitramine explosive such as HMX) integrated with MWCNT/Al/CuO arrays. The improvement of the thermal conductivity is due to the presence of nano MWCNT with 
metal/metal oxide resulted in more pyrolysis products which activated the molecules and promoted the RDX decomposition, as a result the activation energy was decreased.

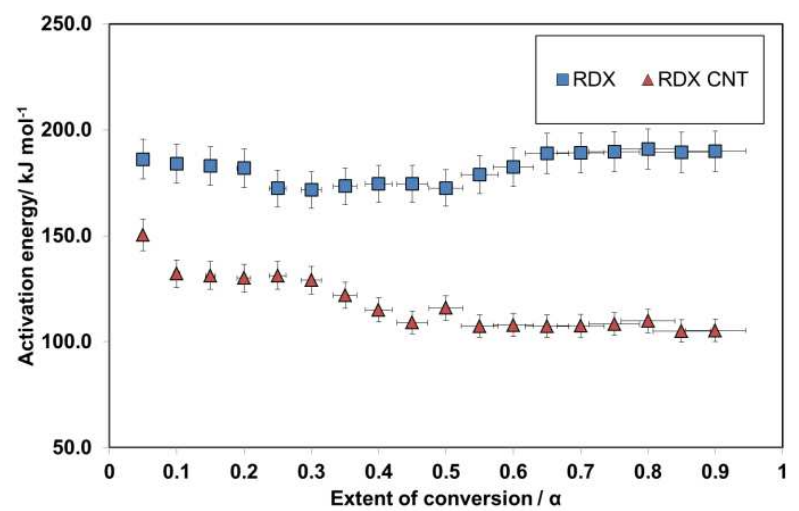

Fig. 10: Dependence of activation energies of RDX/MWCNT/Al/CuO and RDX on the conversion extend by modified KAS method.

\section{Conclusion}

The field of nanoscale thermite reactions is a promising new field of research especially with the energetic materials. The MWCNTs was successfully catalysed with pre-treated surface in two main stages. The $\mathrm{CuO}$ was well metalized the catalysed MWCNTs and achieved good dispersion of with $\mathrm{Al}$ and RDX. The used TEM clearly displays the dispersed thermite in the catalysed MWCNTs. Regarding to the thermogravimetric analysis (TG), it was concluded that the addition of nano-thermite to RDX have the same decomposition as the pure RDX. However, the kinetics of decomposition of nano-thermite composite was changed and decreased the activation energy. The activation energy of the nano-thermite is $135 \mathrm{~kJ} \mathrm{~mol}^{-1}$ and is lower than the RDX $\left(181.6 \mathrm{~kJ} \cdot \mathrm{mol}^{-1}\right)$. The activation energy of the studied nanothermite composite, decreased along with the extent of conversion of 0.55 then started to be on the same value till the end of decomposition. The thermal stability considered constant after the addition of the nano-thermite to the composite. The nano-thermite composite seems to enhance the performance due to presence of the Alex and $\mathrm{CuO}$ with high stability after their addition to the RDX and could be used for practical military and civilian applications.

\section{References}

[1] T.M. Klapötke, Chemistry of high-energy materials, Walter de Gruyter GmbH \& Co KG, 2015.

[2] S. Zeman, M. Jungová, Sensitivity and performance of energetic materials, Propellants, Explosives, Pyrotechnics, 41 (2016) 426-451. 
[3] M. Comet, C. Martin, F. Schnell, D. Spitzer, Nanothermites: a short review. factsheet for experimenters, present and future challenges, Propellants, Explosives, Pyrotechnics, 44 (2019) 18-36.

[4] S. Fischer, M. Grubelich, A survey of combustible metals, thermites, and intermetallics for pyrotechnic applications, in: 32nd Joint Propulsion Conference and Exhibit, 1996, pp. 3018.

[5] L.T. DeLuca, Overview of Al-based nanoenergetic ingredients for solid rocket propulsion, Defence Technology, 14 (2018) 357-365.

[6] C. Hou, X. Geng, C. An, J. Wang, W. Xu, X. Li, Preparation of Al nanoparticles and their influence on the thermal decomposition of RDX, Central European Journal of Energetic Materials, 10 (2013).

[7] A. Elbeih, S. Zeman, Characteristics of melt cast compositions based on cis-1, 3, 4, 6tetranitrooctahydroimidazo-[4, 5 d] imidazole $(\mathrm{BCHMX}) / \mathrm{TNT}$, Central European Journal of Energetic Materials, 11 (2014).

[8] H. Ritter, S. Braun, High explosives containing ultrafine aluminum ALEX, Propellants, Explosives, Pyrotechnics, 26 (2001) 311-314.

[9] N.H. Yen, L.Y. Wang, Reactive metals in explosives, Propellants, Explosives, Pyrotechnics, 37 (2012) 143-155.

[10] M.A. Machado, D.A. Rodriguez, Y. Aly, M. Schoenitz, E.L. Dreizin, E. Shafirovich, Nanocomposite and mechanically alloyed reactive materials as energetic additives in chemical oxygen generators, Combustion and Flame, 161 (2014) 2708-2716.

[11] R.N. Rogers, Thermochemistry of explosives, Thermochimica Acta, 11 (1975) 131-139.

[12] T. Elshenawy, S. Soliman, A. Hawass, High density thermite mixture for shaped charge ordnance disposal, Defence technology, 13 (2017) 376-379.

[13] S. Fischer, M. Grubelich, A survey of combustible metals, thermites, and intermetallics for pyrotechnic applications, in: 32nd Joint Propulsion Conference and Exhibit, American Institute of Aeronautics and Astronautics, 1996.

[14] S. Elbasuney, M.G. Zaky, M. Radwan, S.F. Mostafa, Stabilized super-thermite colloids: A new generation of advanced highly energetic materials, Applied Surface Science, 419 (2017) 328-336.

[15] A.M.A. M. Gaber Zaky, Rakesh P. Sahu, Ishwar K. Puri, Mostafa, S.E. Radwan, Nanothermite colloids: A new prospective for enhanced performance, Defence Technology, 15 (2019) 319-325.

[16] I. Monk, M. Schoenitz, R. Jacob, E. Dreizin, M. Zachariah, Combustion characteristics of stoichiometric $\mathrm{Al}-\mathrm{CuO}$ nanocomposite thermites prepared by different methods, Combustion Science and Technology, 189 (2017) 555-574.

[17] X. Chen, J. Xia, J. Peng, W. Li, S. Xie, Carbon-nanotube metal-matrix composites prepared by electroless plating, Composites Science and Technology, 60 (2000) 301-306.

[18] A. Peigney, C. Laurent, E. Flahaut, R. Bacsa, A. Rousset, Specific surface area of carbon nanotubes and bundles of carbon nanotubes, Carbon, 39 (2001) 507-514.

[19] Q.-L. Yan, M. Gozin, F.-Q. Zhao, A. Cohen, S.-P. Pang, Highly energetic compositions based on functionalized carbon nanomaterials, Nanoscale, 8 (2016) 4799-4851.

[20] S. Arai, M. Endo, Carbon nanofiber-copper composite powder prepared by electrodeposition, Electrochemistry communications, 5 (2003) 797-799.

[21] S. Arai, M. Endo, N. Kaneko, Ni-deposited multi-walled carbon nanotubes by electrodeposition, Carbon, 42 (2004) 641-644. 
[22] K. Yamagishi, S. Yae, N. Okamoto, N. Fukumuro, H. Matsuda, Adsorbates formed on non-conducting substrates by two-step catalyzation pretreatment for electroless plating, Hyomen Gijutsu(Journal of the Surface Finishing Society of Japan), 54 (2003) 150-154.

[23] S.I. Cha, K.T. Kim, S.N. Arshad, C.B. Mo, S.H. Hong, Extraordinary strengthening effect of carbon nanotubes in metal-matrix nanocomposites processed by molecular-level mixing, Advanced Materials, 17 (2005) 1377-1381.

[24] W.A. Trzciński, S. Cudziło, L. Szymańczyk, Studies of detonation characteristics of aluminum enriched RDX compositions, Propellants, Explosives, Pyrotechnics, 32 (2007) 392-400.

[25] P. Sahoo, S.K. Das, Tribology of electroless nickel coatings-a review, Materials \& Design, 32 (2011) 1760-1775.

[26] K. Chin, A. Gohel, W. Chen, H. Elim, W. Ji, G. Chong, C. Sow, A. Wee, Gold and silver coated carbon nanotubes: an improved broad-band optical limiter, Chemical Physics Letters, 409 (2005) 85-88.

[27] A.A. Sahraei, H.N. Saeed, A. Fathi, M. Baniassadi, S.S. Afrookhteh, M.K. Besharati Givi, Formation of homogenous copper film on MWCNTs by an efficient electroless deposition process, Science and Engineering of Composite Materials, 24 (2017) 345-352.

[28] A. Maqbool, M.A. Hussain, F.A. Khalid, N. Bakhsh, A. Hussain, M.H. Kim, Mechanical characterization of copper coated carbon nanotubes reinforced aluminum matrix composites, Materials characterization, 86 (2013) 39-48.

[29] D.G. Piercey, T.M. Klapoetke, Nanoscale aluminum-metal oxide (thermite) reactions for application in energetic materials, Central European Journal of Energetic Materials, 7 (2010) 115-129.

[30] S. Chatterjee, U. Deb, S. Datta, C. Walther, D.K. Gupta, Common explosives (TNT, RDX, HMX) and their fate in the environment: Emphasizing bioremediation, Chemosphere, 184 (2017) 438-451.

[31] A. Elbeih, A.K. Hussein, T. Elshenawy, S. Zeman, S.M. Hammad, A. Baraka, M.A. Elsayed, M. Gobara, H. Tantawy, Enhancing the explosive characteristics of a Semtex explosive by involving admixtures of BCHMX and HMX, Defence Technology, 16 (2020) 487-492.

[32] C.M. Mahoney, A.J. Fahey, K.L. Steffens, B.A. Benner Jr, R.T. Lareau, Characterization of composition $\mathrm{C} 4$ explosives using time-of-flight secondary ion mass spectrometry and X-ray photoelectron spectroscopy, Analytical chemistry, 82 (2010) 7237-7248.

[33] M.D. Joseph, S.K. Jangid, R.S. Satpute, B.G. Polke, T. Nath, S.N. Asthana, A.S. Rao, Studies on advanced RDX/TATB based low vulnerable sheet explosives with HTPB binder, Propellants, Explosives, Pyrotechnics: An International Journal Dealing with Scientific and Technological Aspects of Energetic Materials, 34 (2009) 326-330.

[34] T. Elshenawy, A. Elbeih, Q.M. Li, A modified penetration model for copper-tungsten shaped charge jets with non-uniform density distribution, Central European Journal of Energetic Materials, 13 (2016).

[35] S. Arai, M. Endo, S. Hashizume, Y. Shimojima, Nickel-coated carbon nanofibers prepared by electroless deposition, Electrochemistry communications, 6 (2004) 10291031.

[36] S.-M. Bak, K.-H. Kim, C.-W. Lee, K.-B. Kim, Mesoporous nickel/carbon nanotube hybrid material prepared by electroless deposition, Journal of Materials Chemistry, 21 (2011) 1984-1990. 
[37] M. Jagannatham, S. Sankaran, H. Prathap, Electroless nickel plating of arc discharge synthesized carbon nanotubes for metal matrix composites, Applied Surface Science, 324 (2015) 475-481.

[38] L.-M. Ang, T.A. Hor, G.-Q. Xu, C.-h. Tung, S. Zhao, J.L. Wang, Electroless plating of metals onto carbon nanotubes activated by a single-step activation method, Chemistry of Materials, 11 (1999) 2115-2118.

[39] F. Wang, S. Arai, M. Endo, The preparation of multi-walled carbon nanotubes with a NiP coating by an electroless deposition process, Carbon, 43 (2005) 1716-1721.

[40] S. Vyazovkin, A.K. Burnham, J.M. Criado, L.A. Pérez-Maqueda, C. Popescu, N. Sbirrazzuoli, ICTAC Kinetics Committee recommendations for performing kinetic computations on thermal analysis data, Thermochimica Acta, 520 (2011) 1-19.

[41] R. Svoboda, J. Málek, Is the original Kissinger equation obsolete today?, Journal of Thermal Analysis and Calorimetry, 115 (2014) 1961-1967.

[42] A.K. Hussein, A. Elbeih, S. Zeman, Thermal decomposition kinetics and explosive properties of a mixture based on cis-1, 3, 4, 6-tetranitrooctahydroimidazo-[4, 5-d] imidazole and 3-nitro-1, 2, 4-triazol-5-one (BCHMX/NTO), Thermochimica Acta, 655 (2017) 292-301.

[43] M. Abd-Elghany, T.M. Klapötke, A. Elbeih, Investigation of 2, 2, 2-trinitroethylnitrocarbamate as a high energy dense oxidizer and its mixture with Nitrocellulose (thermal behavior and decomposition kinetics), Journal of Analytical and Applied Pyrolysis, 128 (2017) 397-404.

[44] U. Nair, S. Asthana, A.S. Rao, B. Gandhe, Advances in high energy materials, Defence Science Journal, 60 (2010) 137.

[45] M.W. Beckstead, K. Puduppakkam, P. Thakre, V. Yang, Modeling of combustion and ignition of solid-propellant ingredients, Progress in Energy and Combustion Science, 33 (2007) 497-551.

[46] A.K. Hussein, A. Elbeih, S. Zeman, The effect of glycidyl azide polymer on the stability and explosive properties of different interesting nitramines, RSC advances, 8 (2018) 17272-17278.

[47] M. Abd-Elghany, A. Elbeih, T.M. Klapötke, Thermo-analytical study of 2, 2, 2 trinitroethyl-formate as a new oxidizer and its propellant based on a GAP matrix in comparison with ammonium dinitramide, Journal of Analytical and Applied Pyrolysis, 133 (2018) 30-38.

[48] A. Elbeih, M. Abd-Elghany, T.M. Klapötke, Kinetic parameters of PBX based on Cis-1, 3, 4, 6-tetranitroocta-hydroimidazo-[4, 5-d] imidazole obtained by isoconversional methods using different thermal analysis techniques, Propellants, Explosives, Pyrotechnics, 42 (2017) 468-476.

[49] M. Abd-Elghany, T.M. Klapötke, A. Elbeih, Thermal behavior and decomposition kinetics of Bis (2, 2, 2-trinitroethyl)-oxalate as a high energy dense oxidizer and its mixture with nitrocellulose, Propellants, Explosives, Pyrotechnics, 42 (2017) 1373-1381.

[50] A.K. Hussein, S. Zeman, A. Elbeih, Thermo-analytical study of glycidyl azide polymer and its effect on different cyclic nitramines, Thermochimica Acta, 660 (2018) 110-123.

[51] M. Abd-Elghany, T.M. Klapötke, A. Elbeih, S. Hassanein, T. Elshenawy, Study of thermal reactivity and kinetics of HMX and its PBX by different methods, Chin J Exploss Propellants, 2 (2017) 24-32.

[52] Q.-L. Yan, F.-Q. Zhao, K.K. Kuo, X.-H. Zhang, S. Zeman, L.T. DeLuca, Catalytic effects of nano additives on decomposition and combustion of RDX-, HMX-, and AP- 
based energetic compositions, Progress in Energy and Combustion Science, 57 (2016) $75-136$.

[53] C. Zhang, Q. Peng, L. Wang, X. Wang, Thermal Sensitivity of HMX Crystals and HMXBased Explosives Treated under Various Conditions, Propellants, Explosives, Pyrotechnics, 35 (2010) 561-566.

[54] Z. Jiang, S.-F. Li, F.-Q. Zhao, P. Chen, C.-M. Yin, S.-W. Li, Effect of nano metal powder on the thermal decomposition characteristics of HMX, Tuijin Jishu, Journal of Propulsion Technology, 23 (2002) 258-261. 